\title{
A heterogeneous approach for modelling blood flow in an arterial segment
}

\author{
A. Di Carlo ${ }^{1}$, P. Nardinocchi ${ }^{2}$, G. Pontrelli ${ }^{3}$, L. Teresi ${ }^{1}$ \\ 'Dipartimento di Ingegneria Civile \\ Università di "Roma Tre", Roma, Italy \\ ${ }^{2}$ Dipartimento di Ingegneria Strutturale e Geotecnica \\ Università di Roma "La Sapienza", Roma, Italy \\ ${ }^{3}$ Istituto per le Applicazioni del Calcolo \\ CNR - Roma, Italy
}

\begin{abstract}
A differential model of blood flow through an arterial vessel is presented. The flow equations averaged across the wall thickness are in conjunction with the equation of the compliant wall modelled as an elastic axisymmetric membrane. Such onedimensional model describing the nonlinear fluid-wall interaction is coupled with a six compartment lumped parameter model which accounts for the global circulatory features.

The full nonlinear equations are approximated by using a numerical finite difference method on a staggered grid. Some numerical simulations show the characteristics of the wave propagation and the dependence on the relevant parameters.
\end{abstract}

\section{Introduction}

Mathematical models for cardiovascular system are largely used to simulate blood flow in arteries and to predict dynamical patterns in physiological and pathological conditions. Due to their complexity, comprehensive models are difficult to be settled. Complexity derives from geometry, time dependence and mechanical properties of the wall [1].

According to the specific scale of the phenomenon to be studied, various degrees of simplification at some levels have been proposed. One of them concerns the 
physical dimension of the model. In lumped parameters models, different complex regions of the vascular system are collected in simpler compartments and connected to form a closed loop, in analogy with the electrical circuits. The number of blocks is related with the desired degree of detail. Many lumped parameter models (also called 0D models) have been devised for the full cardiovascular system and for specific vascular districts [2]. The main advantage of such an approach is the possibility to model the circulatory system and the blood pressure-velocities relationship with a relatively simple and computationally effective model. They provide the evolution of the mean flow variables but do not give any information on the mechanical fluid-wall interaction.

When wave propagation phenomena are of interest, one-dimensional models are commonly used. They constitute a higher degree of approximation and, based on the hypothesis that one spatial dimension is prevailing on the others, are obtained by averaging the flow motion equation over the cross section. In this work a simple one-dimensional model for the fluid-structure problem describing the blood flow and the wave propagation in an arterial segment is presented. The wall is modelled as an axisymmetric membrane that deforms in both radial and longitudinal directions under the forces exerted by the fluid. The wall constitutive equation for the vascular tissue proposed in [3] has been used.

To take advantage of the 1D model and not give up to a simple description of the systemic circulation, the idea of coupling systems of different physical dimensions has been recently developed (multiscale model) [4] and is reproposed here. The matching interface conditions between a $0 \mathrm{D}$ model and the $1 \mathrm{D}$ model inserted at the level of descending aorta are addressed. Many computational results show the effectiveness of such heterogeneous approach and point out the dependence on the physical parameters.

\section{The wall-fluid interaction}

The blood flowing in a compliant vessel is a complex dynamical system and constitutes a genuine fluid-structure problem. The fluid motion and the wall deformation are mutually influenced and their coupling is responsible for effects which cannot be explained by each of them alone. When wave propagation phenomena are concerned, simplified models for the system "blood-arterial wall" can be devised. In particular, due to the small deformations of the vascular wall and to the unidirectional nature of blood flow, a one-dimensional model is adopted.

\subsection{The flow equations}

Let us consider a homogeneous fluid of density $\rho$ and of viscosity $\mu$, flowing in a straight, axisymmetric, distensible tube of circular cross section.

The quasi-1D cross averaged momentum equation is [1]

$$
\frac{\partial u}{\partial t}+u \frac{\partial u}{\partial x}=-\frac{1}{\rho} \frac{\partial p}{\partial x}+f
$$


where $x$ is the axial coordinate, $u$ is the axial velocity, $p$ the transmural pressure, both averaged over the cross section, and $t$ denotes the time. The viscous term $f$ is approximated by the friction term of the Poiseuille steady flow in a tube of radius $R$ :

$$
f \simeq-\frac{8 \mu u}{\rho R^{2}} .
$$

As a consequence, the wall shear stress is given by

$$
\tau=\left.\frac{d u}{d r}\right|_{R} \simeq-\frac{4 \mu u}{\rho R} .
$$

Strictly speaking, the expressions (2) and (3) hold for a steady flow in a rigid tube, but they are considered acceptable for quasi steady flows and for small deformations.

The principle of conservation of mass in a deformable tube is expressed by the following the continuity equation [1]

$$
\frac{\partial R}{\partial t}+\frac{R}{2} \frac{\partial u}{\partial x}+u \frac{\partial R}{\partial x}=0
$$

\subsection{The wall equations}

The vessel wall is modelled as an elastic axisymmetric membrane, that is a $2 \mathrm{D}$ thin shell with a mass negligible (wall thickness $\rightarrow 0$ ) compared with that of the fluid contained in it. The membrane, which has no bending stiffness, is capable to deform under the forces exerted by the fluid (i.e. the shear stress $\tau$ - cfr. (3) - and the transmural pressure $p)$. Let $\left(x_{P}(s), r_{P}(s)\right)$ be the Lagrangian coordinates of a particle $P$ with $s$ a parametric coordinate along the membrane in its symmetry plane. In such reference frame, the principal deformation ratios in the meridional and circumferential directions are respectively

$$
\lambda_{1}=\sqrt{\left(\frac{d r_{P}}{d s}\right)^{2}+\left(\frac{d x_{P}}{d s}\right)^{2}}, \quad \lambda_{2}=\frac{r_{P}}{R^{*}},
$$

where $R^{*}$ is the undeformed radius (corresponding to the zero transmural pressure).

Since the fluid equations are expressed in Eulerian coordinates, let us operate a transformation of coordinate and let us indicate by $R(x, t)$ and $S(x, t)$ the Eulerian counterparts of the Lagrangian coordinates of a particle of the a membrane (see [5]). In such coordinate system, the stretches (5) are written as

$$
\lambda_{1}=\sqrt{\frac{1+R^{\prime 2}}{S^{\prime 2}}}, \quad \lambda_{2}=\frac{R}{R^{*}}
$$

(the prime denoting $x$-derivative). The membrane equilibrium equations in tangential and normal directions are provided [5]

$$
R^{\prime}\left(T_{1}-T_{2}\right)+R T_{1}^{\prime}=\rho \tau R\left(1+R^{\prime 2}\right)^{\frac{1}{2}}
$$




$$
\frac{-R^{\prime \prime}}{\left(1+R^{\prime 2}\right)^{\frac{3}{2}}} T_{1}+\frac{1}{R\left(1+R^{\prime 2}\right)^{\frac{1}{2}}} T_{2}=p .
$$

Let us now introduce a constitutive equation for the arterial vessel that give an expression for $T_{1}$ and $T_{2}$ in the equations (7). For an incompressible hyperelastic material, it is possible to define a strain-energy function $W$ as a function of the principal strains: it represents the elastic stored energy per unit volume in terms of the strain variables and is a potential for the stress.

A constitutive strain-energy density function $w$ modelling the mechanical properties of the arterial wall has been recently proposed by Zhou \& Fung [3] as

$$
w=c_{0}\left(e^{Q}-1\right), \quad Q=c_{1} E_{1}^{2}+c_{2} E_{2}^{2}+2 c_{3} E_{1} E_{2},
$$

where $c_{0}$ is a material parameter having the dimensions of dyne $/ \mathrm{cm}, c_{1}, c_{2}$, $c_{3}$ are nondimensional constants (with $c_{1} \approx c_{2}$ and $c_{1}, c_{2} \gg c_{3}$ ) and $E_{k}=$ $\frac{1}{2}\left(\lambda_{k}^{2}-1\right), k=1,2$ are the principal Green strains. Once the form of $w$ is specified, the mechanical properties are completely determined, being the stress components (averaged across the thickness) along the longitudinal and circumferential directions given by differentiation of $w$

$$
\begin{aligned}
& T_{1}\left(\lambda_{1}, \lambda_{2}\right)=\frac{\lambda_{1}}{\lambda_{2}} \frac{\partial w}{\partial E_{1}}=\frac{1}{\lambda_{2}} \frac{\partial w}{\partial \lambda_{1}}=2 \frac{\lambda_{1}}{\lambda_{2}} c_{0} e^{Q}\left(c_{1} E_{1}+c_{3} E_{2}\right), \\
& T_{2}\left(\lambda_{1}, \lambda_{2}\right)=\frac{\lambda_{2}}{\lambda_{1}} \frac{\partial w}{\partial E_{2}}=\frac{1}{\lambda_{1}} \frac{\partial w}{\partial \lambda_{2}}=2 \frac{\lambda_{2}}{\lambda_{1}} c_{0} e^{Q}\left(c_{3} E_{1}+c_{2} E_{2}\right) .
\end{aligned}
$$

The former relations hold in the case of an anisotropic membrane, wherein principal directions of strain and stress coincide and express the property that the instantaneous Young's modulus increases with the strain, but with a different amount in the two directions.

\section{Boundary conditions and lumped parameter models}

The full nonlinear system fluid-structure is modelled by the coupled equations (1), (4), (7) (with the replacements (6), (9), (10)) and a numerical method will be used. The analysis of the linearized problem in the neighbourhood of a stressed configuration will be the object of a forthcoming work [6]. The above differential equations have to be solved in a finite domain representing an arterial segment. Such a segment is extracted from the arterial tree and boundary conditions of physical significance for the variables are required. To this aim, the presence of the remaining vascular bed has to be considered. In [7], a pulsatile pressure is assigned at the inlet as a forcing, and a simple Windkessel 3-element parameter model for the termination is proposed. However, when balance of flows and pressures for the systemic circulation have to be taken into account, models for the closed-loop system should be addressed. They are built by partitioning the whole vascular tree in elementary districts and by "lumping" the dynamical variables in each of them (lumped parameter models). These models date back to the pioneeristic works of 


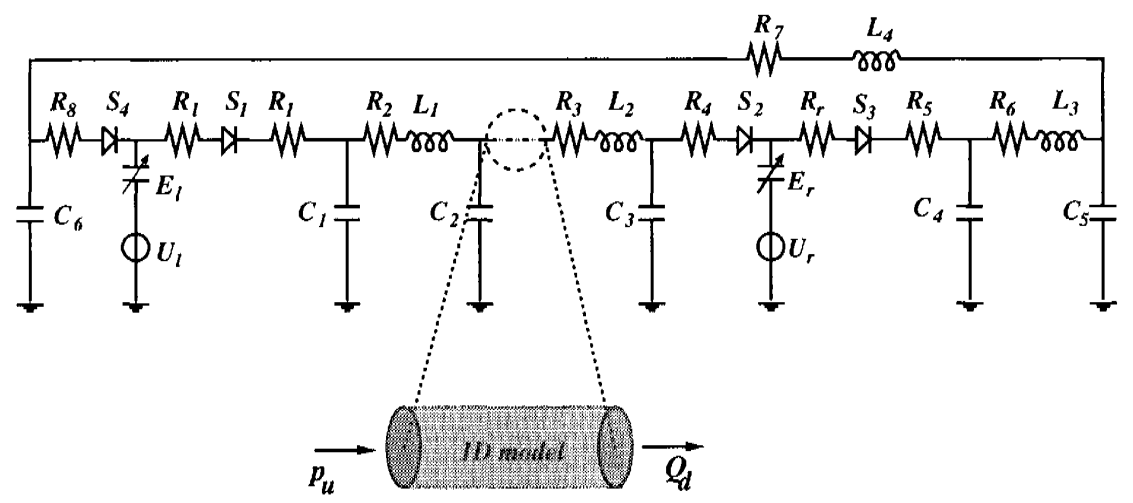

Figure 1: The electrical network analogue to a lumped parameter model at six compartments for the human circulatory system (see Avanzolini et al. [2]) and its coupling with the ID model at the level of the descending aorta. Pressure and flow variables are exchanged at the interface points to guarantee continuity (by courtesy of Formaggia et al. [4]).

Westerhof et al. and are based on the analogy between hydraulic networks and electrical circuits [8]. In the network proposed by Avanzolini et al. [2] for the circulatory system, six sections can be recognized (fig. 1). In each compartment the values of the resistance, compliance and inertance are constant and a linear relationship between flow and pressure is given [8]. These elementary blocks are linked between them and connected with the heart pump to form a closed loop representing the cardiovascular system.

By setting conservation of pressure and of flow in all nodes of the network, a differential linear system

$$
\frac{d X}{d t}=A(t)+f(t, X)
$$

describing the time evolution of the mean values of the variables $X=\left(p_{i}, Q_{i}\right)$ in each compartment is obtained [2].

To account for a comprehensive system of the global circulation, the lumped model (a) and the distributed model (b) presented in the section 2 are coupled. This approach allows to implicitly assign boundary conditions for the system (b). Actually these are easily expressed as a functions of variables of (a) to guarantee the continuity of flow and pressure at the interfaces. Following [4], we have inserted the model (a) in the point of network corresponding to the descending aorta (fig. 1).

The coupled system is equivalent to a 1D model for the full circulatory system where, except for a segment, the remaining arterial tree has been truncated and lumped in a finite number of blocks. On the other way around, the coupled model can be regarded as a lumped parameter model where a compartment has been 
expanded in a distributed model. The connected subsystems (a) $+(b)$ form a unique closed-loop and no boundary condition for the flow variables is required.

If the coupling strategy eliminates the drawback of assigning a boundary value for $u$ and $p$, wall displacement conditions at the extrema of the compliant vessel have to be provided. These are given by considering a long (i.e. of length much larger than the reference radius $R^{*}$ ) vessel with free ends. Therefore the conditions

$$
R^{\prime}=R^{\prime \prime}=0, \quad S^{\prime}=1
$$

hold at the ends. Such conditions imply $\lambda_{1}=1$ (null axial strain - see (6)). From (7.2) it follows that the implicit relation for $R$

$$
R p=T_{2}
$$

(law of Laplace) is prescribed at the boundaries. Moreover, the boundary conditions on $S$

$$
S(0, t)=0, \quad S(L, t)=L^{*}
$$

expressing a finite axial deformation are imposed.

Thus, we solve the differential system (11) and the partial differential system (1),(4),(7), together with interface continuity conditions. Details of the coupling algorithm can be found in [6].

\section{Numerical results and discussion}

To handle the 1D fluid-structure model numerically, the equations (1)-(4)-(7) are solved simultaneously in a finite interval $[0, L]$. Let us consider a sequence of $n+1$ equispaced grid points $\left(x_{i}\right)_{i=0, \ldots, n}$ with $x_{0}=0$ and $x_{n}=L$. The spatial discretization is obtained by evaluating membrane strains and stresses (see eqns. (6), (9), (10)) at $n$ inner points $\xi_{i}=\frac{x_{i}+x_{i+1}}{2}$ of a staggered grid by considering averaged neighboring variables. On the other hand, wall-fluid equilibrium equations (7) and flow equations (1)-(4) are computed at the $n-1$ inner points $x_{i}$ [9]. In the following numerical experiments, the spatial mesh has been obtained by dividing the length of the vessel $L=8 \mathrm{~cm}$ in 800 equal parts $(\Delta x=0.01 \mathrm{~cm}$ ) and with a time step $\Delta t=10^{-4} \mathrm{~s}$. The $1 \mathrm{D}$ model is inserted in correspondence of the descending aortic artery (fig. 1) and is solved coupled with the 0D model. The Runge-Kutta scheme of second order has been used in both the distributed and the lumped parameter model to advance in time. The choice of the above numerical parameters guarantees stability and grid independence. The resulting nonlinear system is solved by a globally convergent Newton type method.

The following numerical values for the distributed model are used [3]:

$$
\begin{array}{lcr}
c_{1}=0.38 & c_{2}=0.26 & c_{3}=0.046 \\
R^{*}=0.8 \mathrm{~cm} & L^{*}=L=8 \mathrm{~cm} & \rho=1.05 \mathrm{~g} / \mathrm{cm}^{3}
\end{array}
$$

In large vessels, as that considered here, the frictional force due to the fluid viscosity is comparatively small and will be neglected $(\mu=0)$. The value of $c_{0}$ is 

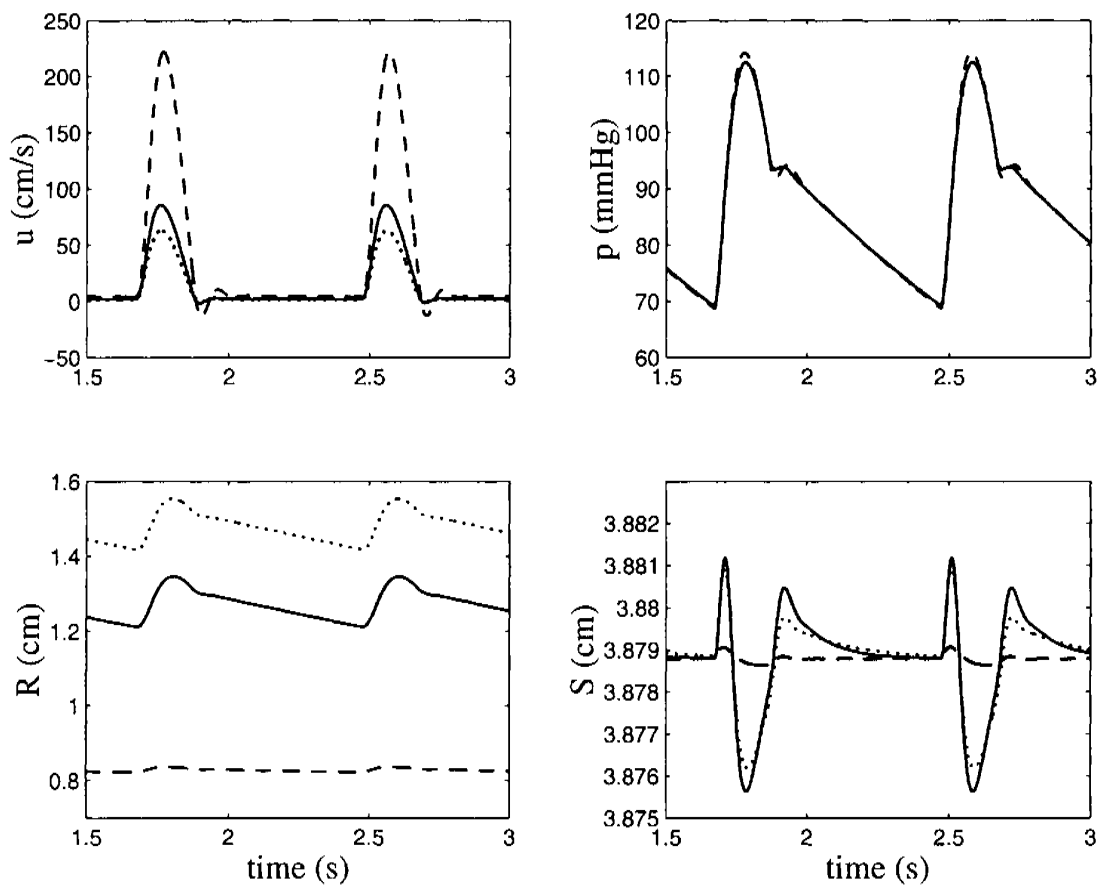

Figure 2: Time histories for $u, p, R, S$ at the center of the artery for three values of the elasticity coefficient $c_{0}\left(c_{0}=10^{5} \mathrm{dyne} / \mathrm{cm}\right.$ dotted line, $c_{0}=$ $2 \cdot 10^{5}$ dyne $/ \mathrm{cm}$ continuous line, $c_{0}=5 \cdot 10^{6}$ dyne $/ \mathrm{cm}$ dashed line).

varied in the range $c_{0}=10^{5}-10^{7}$ dyne $/ \mathrm{cm}$ (note that $c_{0}$ in (8) is obtained by integration across the wall thickness of the analogous density energy function in Zhou and Fung [3]). For a lower value of $c_{0}$ the vessel wall undergoes large deformations that cannot be adequately represented by the present model. For $c_{0} \gg 10^{7}$ the solution approaches to that relative of a rigid tube (see below). Actually, the values of $R^{*}, p$ and $c_{0}$ cannot be chosen independently, but should satisfy a compatibility condition, being $c_{0}$ approximatively equal to $p R$ (mean values), in the linear case. The lumped parameters have been taken from [2].

Subject to a positive heart pressure, transmitted through the $0 \mathrm{D}$ model, the wall expands and oscillates periodically between a maximum and a minimum limits. Similarly, all the flow variables have a periodical behaviour. Actually we recognize a mean value and small superposed fluctuations over it. Such values depend on the elasticity coefficient $c_{0}$ and on the undeformed radius $R^{*}$. In fig. 2 the behaviour of the four variables $u, p, R, S$ in the mid point are depicted for three different values of the parameter $c_{0}$. A small phase lead of $p$ on $u$ is present (see also fig. 4). For larger $c_{0}$ the wall becomes stiffer: as expected, both the radial and longitudinal deformations decrease with $c_{0}$, being the latter comparatively smaller. Despite no significant variation in the pressure is present (a rise of the systolic peak 

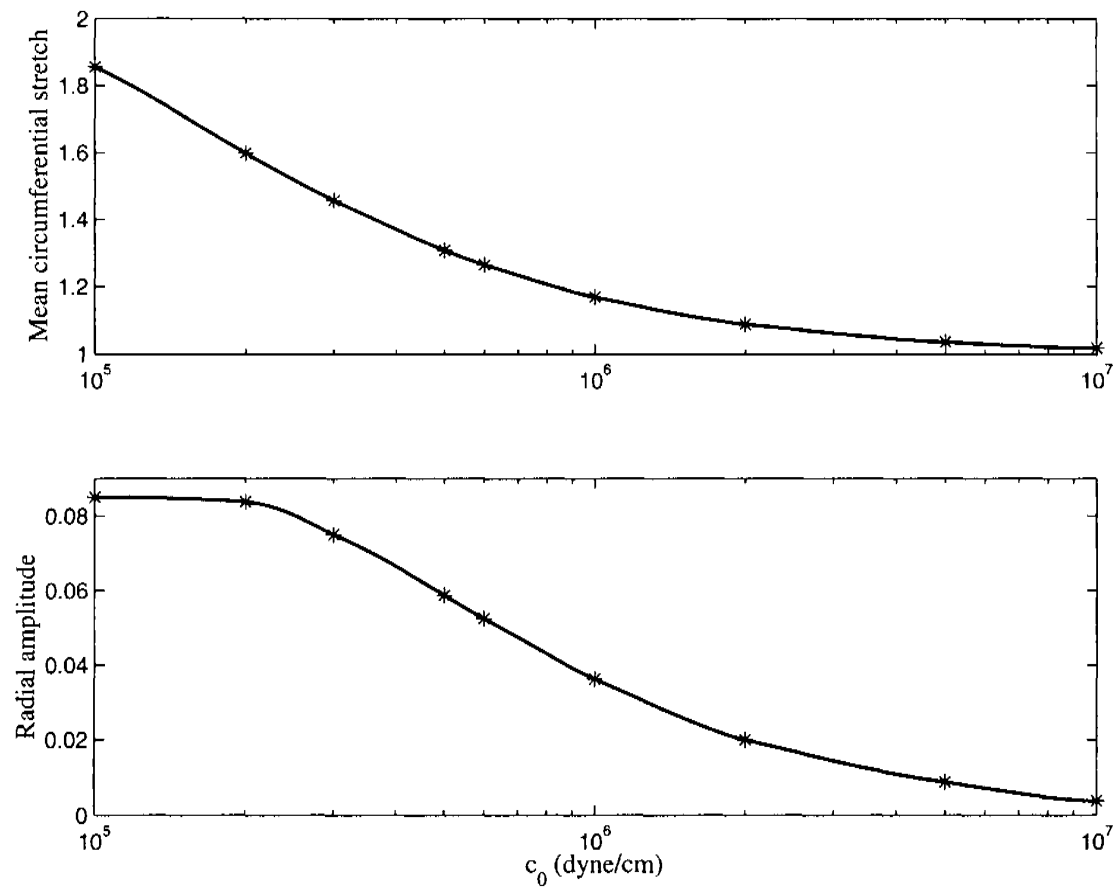

Figure 3: Mean circumferential stretch $\hat{\lambda}_{2}$ (above) and nondimensional radial amplitude $\hat{A}$ (bottom) at the center of the vessel as a function of $c_{0}$. Starred points are results from simulations, continuous curves are obtained by a cubic interpolation. Note the different order of magnitude.

of the pressure is obtained only at large $c_{0}$ ), a sharp increase of the flow velocity is reported, correspondent to the reduced arterial lumen. Some extra oscillations after the systolic peak may be present.

To measure the influence of $c_{0}$ on the radial deformation, let us introduce the mean deformation - referred to the central point $(x=4)$ and computed over the last two periods - as:

$$
\hat{R}=\frac{R_{\max }+R_{\min }}{2}
$$

the mean circumferential stretch $\hat{\lambda}_{2}=\frac{\hat{R}}{R^{*}}$, and the nondimensional radial amplitude $\hat{A}=\frac{R_{\max }-\hat{R}}{R^{*}}$. Both $\hat{\lambda}_{2}$ and $\hat{A}$ drop (the former of $45 \%$, the latter of $95 \%$ ) with $c_{0}$ in the range considered, until an asymptotic value (fig. 3 ).

The velocity of the wave can be obtained by fixing two points in the vessel and measuring the crossing time of a peak. However, such a procedure is not accurate over a short length and for the time and space steps as those considered in this work. Moreover the profiles change their shape as they travel, and it is difficult to 


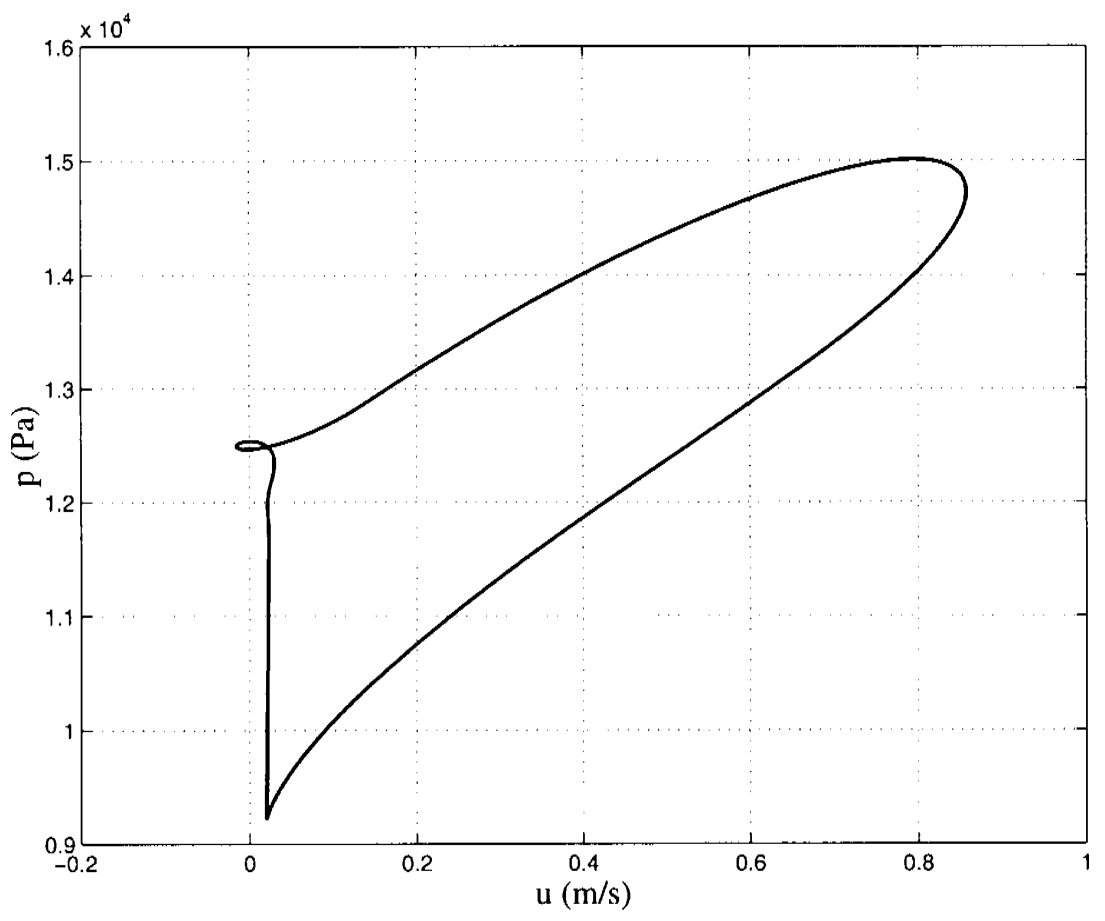

Figure 4: PU loop curve for $c_{0}=2 \cdot 10^{5} \mathrm{dyne} / \mathrm{cm}$ in the central point of the vessel. Slopes of such a curve indicates the local wave speed. A phase lag between the two variables is present.

follow a profile in time. As a consequence, the computed speed value measured for $u, p$ and $R$ between the same grid points may be different, and varies in time. For $c_{0}=2 \cdot 10^{5} d y n e / \mathrm{cm}$, an averaged value of the speed for $u$ wave is found of about $6-7 \mathrm{~m} / \mathrm{s}$.

Khir and Parkers [10] suggest another method to measure the wave speed $\gamma$ in elastic tubes in absence of reflected waves. It is based on the validity of the waterhammer equation $d p= \pm \rho \gamma d u$ and consists in measuring the slope of the PU loop curves. For a typical PU loop as that displayed in fig. 4 , the local wave speed is $\gamma=5.2 \mathrm{~m} / \mathrm{s}$, in agreement with experiments.

\section{Conclusions}

The dynamics of the blood flow in an arterial segment has been studied in relation to the elastic nonlinear properties of the vessel wall. The mechanical fluid-wall interaction is described by a 1D model expressed by a set of four nonlinear partial differential equations. To account for a global circulation features, the distributed model has been coupled with a lumped parameter model which provide the proper 
boundary conditions by reproducing the correct waveforms entering into the vessel and avoid unphysical reflections. Numerical experiments show the effect of the elastic parameters on the flow and on the wall deformations.

The model, though its limitations, offers a predictive insight in propagation phenomena and can be easily generalized to account for vessel tapering and bending. The model turns out to be sensitive to the distributed and lumped parameters and a more realistic estimate of them should be done on the basis on experiments and clinical data.

\section{Acknowledgment}

This work has been carried out under the partial support of the CNR program Agenzia2000, n. CNRC00A3F1, 2001.

\section{References}

[1] Fung, Y.C., Biomechanics: Circulation (2nd Ed.), Springer-Verlag, New York, 1997.

[2] Avanzolini G., Barbini P., Cappello A., Cevenini G., CADCS simulation of the closed-loop cardiovascular system, Int. J. Biomed. Comput., Vol. 22, pp. $39-49,1988$.

[3] Zhou J., Fung Y.C., The degree of nonlinearity and anisotropy of blood vessel elasticity, Proc. Natl. Acad. Sci. USA, Vol. 94, pp. 14255-14260, 1997.

[4] Formaggia L., Nobile F., Quarteroni A., Veneziani A., Multiscale modelling of the circulatory system: a preliminary analysis, Comput. Visual. Sci., Vol. 2, pp. 75-83, 1999.

[5] Pedrizzetti G., Fluid flow in a tube with an elastic membrane insertion, $J$. Fluid Mech., 375, pp. 39-64, 1998.

[6] Di Carlo A., Nardinocchi P., Pontrelli G., Teresi L., The role of the arterial prestress on blood-flow dynamics, in preparation, 2002.

[7] Pontrelli G., Rossoni E., Numerical modelling of the pressure wave propagation in the arterial flow, submitted, 2002.

[8] Westerhof N., Bosman F., Vries C.D., Noordergraaf A., Analog studies of the human systemic arterial tree, J. Biomech., Vol. 2, pp. 121-143, 1969.

[9] Fletcher C.A., Computational techniques for fluid dynamics, Vol. 2, Springer Ser. Comp. Phys., 1991.

[10] Khir A.W., Parker K.H., Measurements of wave speed and reflected waves in elastic tubes and bifurcations, J. Biomech., Vol. 35, pp. 775-783, 2002. 